Canadian Journal of Animal Science

Canadian

Science

Publishing
Canadian Journal of Animal Science

TYPOLOGIE DES ELEVAGES BOVINS LAITIERS DE LA REGION DE SOUK-AHRAS (ALGERIE)

\begin{tabular}{|r|l|}
\hline Journal: & Canadian Journal of Animal Science \\
\hline Manuscript ID & CJAS-2017-0179.R1 \\
\hline Manuscript Type: & Article \\
\hline Complete List of Authors: & $\begin{array}{l}\text { Yozmane, mania } \\
\text { Mebirouk-Boudechiche, Lamia; Agronomic; } \\
\text { Houd-Chaker, Katina } \\
\text { Abdelmadjid, sade }\end{array}$ \\
\hline Keywords: & Breeding, Cattle, Dairy, Farming practices, Forages \\
\hline & \\
\hline
\end{tabular}

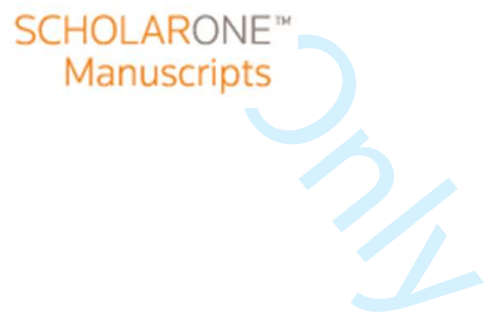

https://mc.manuscriptcentral.com/cjas-pubs 


\title{
Typologie des élevages bovins laitiers de la région de Souk-Ahras (Algérie)
}

\author{
R. Yozmane ${ }^{1}$, L. Mebirouk-Boudechiche ${ }^{2}$, K. Chaker-Houd ${ }^{3}$ et S. Abdelmadjid ${ }^{4}$ \\ ${ }^{1}$ Département de Biologie, Université Badji Mokhtar Annaba, 23000 Algérie
}

\section{yoz ranou@yahoo.fr}

${ }^{2}$ Laboratoire d'épidémio-surveillance, santé, productions et reproduction, expérimentation et thérapie cellulaire des animaux domestiques et sauvages, Université Chadli Bendjedid El

Tarf, B.P 73, 36000, EL Tarf, Algérie.

${ }^{3}$ Laboratoire d'Agriculture et fonctionnement des écosystèmes, Université Chadli Bendjedid El Tarf, B.P 73, 36000, EL Tarf, ALGERIE.

${ }^{4}$ Université Cherif Messaadia de Souk Ahras, 41000, Algérie.

\section{Auteur de correspondance : $\underline{\text { boudechiche.lamia@gmail.com }}$}

\section{Résumé}

L'étude typologique mise en place a pour but de caractériser les différents types d'élevages bovins laitiers dans la wilaya de Souk-Ahras à vocation laitière en Algérie. Pour ceci, nous avons analysé les données d'une enquête qui a porté sur 91 exploitations : analyse des correspondances multiples, suivie d'une Classification ascendante hiérarchique. L'analyse a démontré que le foncier, la taille de cheptel et la race sont les principales caractéristiques qui discriminent les trois groupes identifiés (G1, G2 et G3). Les résultats ont en outre démontré une très faible productivité des élevages malgré l'importance du potentiel génétique du cheptel. Ainsi une surface agricole utile et une taille du cheptel importantes dans les deux premiers groupes (G1 et G2) n'ont pas contribué à l'amélioration de la productivité qui reste similaire au (G3) dans lequel la contrainte foncière est plus accentuée. Cette situation est attribuée principalement à la faible production fourragère en raison de la faible pluviométrie et des surfaces irriguées, réservées principalement aux cultures céréalières jugées plus rentables. Devant ce constat, la forte dépendance envers les approvisionnements en concentré 
dans la ration des vaches peut s'expliquer par les faibles quantités des fourrages disponibles dans les exploitations étudiées.

Mots clés : Algérie, élevage bovin laitier, productivité, typologie, wilaya de Souk-Ahras Titre courant : Caractérisation typologique des élevages bovins laitiers de Souk Ahras

\section{Summary}

The purpose of the typological study is to characterize the different types of dairy cattle farming, in the Wilaya of Souk-Ahras, with a dairy vocation in Algeria. For this, we analyzed data from a survey that covered 91 farms by multiple correspondence analysis, followed by an ascending hierarchical classification. The analysis demonstrated that land, livestock size and race are the main characteristics that discriminate the three identified groups (G1, G2 and G3). The results also showed a very low productivity of the livestock farms despite the importance of the genetic potential of the livestock. Thus, a useful agricultural area and livestock size in the first two groups (G1 and G2) did not contribute to the productivity improvement that is similar to the G3 in which the land constraint is more pronounced. This is mainly attributed to low fourrage production due to low rainfall and irrigated areas, which are mainly reserved for cereal crops considered more profitable. Given this situation, the high dependence on concentrate supplies in the cow's ration can be explained by the low quantities of fourrage available on the farms studied.

Key words: Algeria, dairy cattle breeding, productivity, typology, Souk-Ahras wilaya Running title: Typological characterization of dairy cattle farms in Souk Ahras

\section{INTRODUCTION}

La situation des élevages bovins laitiers en Algérie est particulière du fait que la majeure partie des élevages sont situés dans des zones semi-arides affectées une bonne partie de l'année par la sécheresse. Elever des vaches laitières, grandes consommatrices d'herbe verte 
demeure ainsi une gageure pour le secteur laitier. Par ailleurs, tous les programmes nationaux et dispositifs politiques, instaurés dans le but de construire une filière lait autosuffisante et solide, aspirent à développer l'élevage du bovin laitier (Zaida 2016). Certaines de ces mesures tendent à améliorer le rendement des élevages et impliquent l'intégration de races hautement laitières. En effet le but étant d'augmenter la production et, par là même, de réduire la facture des importations de la poudre de lait qui a couté au pays 800 millions de Dollars en 2016 (CNIS 2016). Il est a noté que la production nationale de lait de vache, se heurte à beaucoup de problèmes de gestion technique. Sur ce point de vue le facteur limitant alimentaire est souvent montré comme la principale contrainte technique des élevages algériens.

Le développement de l'élevage bovin laitier est conditionné par la disponibilité et l'amélioration des ressources fourragères. En Algérie, le déficit hydrique et la faible pluviométrie affectent la production des fourrages. Par ailleurs, la production à tendance à s'orienter de plus en plus vers les cultures spéculatives au détriment des cultures fourragères. L'absence et la cherté des semences fourragères importées, viennent encore pénalisées cette production. En outre, la faible valeur nutritionnelle des fourrages ainsi que leur indisponibilité et cherté rend la ration des vaches fortement tributaire des apports de concentrés, lesquels sont utilisés de façon irrationnelle alourdissant davantage les charges des élevages laitiers. Bouzida et al. (2010) affirment que le niveau d'alimentation des vaches laitières dans les élevages et l'insuffisance de l'offre fourragère sont d'importants facteurs limitants, qui contrarient le développement de la production laitière et de l'élevage algérien. Il faut dire qu'en raison de l'insuffisance de fourrages, la production quotidienne de lait varie entre 15 et 25 litres/vache, alors que les normes internationales oscillent entre 40 et 65 litres $\begin{array}{lll}\text { par } & \text { jour 2016). }\end{array}$ Il est tout aussi important de signaler, le manque d'investissements en matériels mécaniques et en mains d'œuvre dans les élevages laitiers locaux. Ceci malheureuses sont limitées par le 
manque des capitaux pour l'acquisition d'équipent laitiers et fourragers d'une part et d'autre part par le manque de formation et d'accompagnement des éleveurs pour l'acquisition des crédits pour financer leurs projets.

La wilaya de Souk-Ahras est considérée comme l'un des principaux bassins laitiers algérien avec 5500 éleveurs exploitant 50200 vaches laitières dont 9000 de races importées à haut potentiel génétique, 12200 vaches croisées et 29000 vaches de races locales. (DSA 2016). Ce facteur, bien que favorable, n'a toutefois pas permis, dans cette région, le développement de la productivité des élevages qui demeure très insuffisante pour combler les besoins en lait actuels et à venir du pays.

La typologie étant un outil synthétique, pourrait servir de base pour la conception de références communes à tous les intervenants du milieu agricole de manière à améliorer l'efficacité des conseils techniques et de gestion auprès des exploitations (Leblanc 2012). Ceci permet une vision plus globale sur le fonctionnement des exploitations, de cibler leurs besoins et d'améliorer leurs productivités ainsi leurs rentabilités.

C'est dans cette optique que nous avons voulu, à travers d'une analyse typologique, distinguer les types d'exploitations bovins laitiers dans la région d'étude et d'analyser les contraintes qui limitent leurs productivité et d'apporter une contribution pour le développement de la filière lait.

\section{MATERIEL ET METHODES}

\section{Description de la zone d'étude}

La wilaya de Souk-Ahras est située au Nord-Est de l'Algérie, près de la frontière tunisienne (figure 1). Elle s'étend sur une superficie de $4541 \mathrm{~km}^{2}$ avec une altitude moyenne de 1000 $\mathrm{m}$ au Nord et $650 \mathrm{~m}$ au Sud (Agence Nationale d'Intermédiation et de Régulation Foncière 2011). La partie Nord de la région est exposée aux influences climatologiques 
méditerranéennes alors que la partie Sud se caractérise par un climat semi-aride. La wilaya se distingue par un été chaud et sec avec une moyenne de $33{ }^{\circ} \mathrm{C}$ enregistrant des pics de $42{ }^{\circ} \mathrm{C}$ et un hiver froid et humide avec une moyenne de $12{ }^{\circ} \mathrm{C}$. La pluviométrie atteint en moyenne $600 \mathrm{~mm}$ par an (250 $\mathrm{mm}$ au sud et $700 \mathrm{~mm}$ au nord) souvent mal repartie. Tandis que les hauteurs enregistrent une chute de neige importante durant l'hiver (Station météorologique de Souk-Ahras 2016).

Les potentialités hydriques de la Wilaya de Souk-Ahras estimées à 265 Millions de mªn, sont assez importantes pouvant être utilisé pour promouvoir toute spéculation agricole nécessitant le recours à l'irrigation. Elles sont constituées des eaux de surface principalement composées de deux grands Barrages (Ain-Dalia et Foum el Kenga) d'une capacité de 243 Millions de $\mathrm{m}^{3}$ et des eaux souterraines avec 22 Millions de $\mathrm{m}^{3}$.

Avec près de 100000 têtes de bovins dont plus de la moitié est constituée de vaches laitières dont 9000 de races importées principalement composées de la race Holstein et Montbéliarde, 12200 vaches hybrides (croisées) et 29000 vaches de $\mathrm{ra}^{2} \&$ ces locales (Brune d'Atlas), la région de Souk-Ahras est considérée comme le bassin laitier de l'Est du pays avec une production de 50 millions de litres/an (DSA 2016). La wilaya ne compte que deux petites laiteries d'une capacité de 50000 litres, $40 \%$ du volume produit est écoulé auprès de petits commerçants spécialisés dans le lait et ses dérivés, et de cafetiers où la consommation de lait cru de vache reste très appréciée. Le reste est livré à des laiteries situées dans les wilayas limitrophes.

Eu égard aux atouts que possède la wilaya, la mise en œuvre de systèmes fourragers plus performants est tout à fait réalisable malgré les contraintes existantes (manque de semence fourragères, insuffisance dans les investissements hydriques). La surface fourragère (SAF) de la wilaya est très limitée, elle ne présente que 45250 ha comparant à celle de la céréaliculture présentant 137200 ha. La SAF est répartie entre 35000 ha pour le fourrage naturel et 9000 ha 
est présenté essentiellement par l'orge en vert, 250 ha de luzerne, 500 ha de sorgho, 100 ha maïs fourrager et 400 ha de vesce avoine (DSA 2016).

\section{Modalités de l'enquête}

La méthodologie est basée sur un nombre d'enquêtes réalisées auprès des éleveurs laitiers des différentes zones agro écologiques de la wilaya de Souk-Ahras. Pour ceci des enquêtes préliminaires ont été effectuées dans l'ensemble des élevages bovins laitiers afin de déterminer ceux qui feront l'objectif des enquêtes plus approfondies. En effet 91 exploitations ont été sélectionnées durant la campagne agricole 2015-2016 pour l'élaboration d'une analyse typologique. L'échantillonnage a été réalisé sur la base des informations collectées auprès du Groupe d'appui aux éleveurs laitiers (GAPEL) de Souk-Ahras, plus connu sous le nom de « Projet Alban ». Ce dernier est issu d'un projet de coopération technique avec le partenaire français, chargé de l'encadrement technique des éleveurs bovins laitiers dans trois wilayas Algériennes y compris Souk-Ahras englobant ainsi 200 éleveurs adhérents. L'échantillonnage a été fait d'une manière aléatoire tout en respectant certains critères :1) éleveurs adhérés au contrôle laitier pour la disponibilité des informations relatifs à l'enquête ; 2) la motivation de l'éleveur de participer à l'étude ; 3) accès facile ; 4) diversité préalable dans la localisation géographique des élevages.

Pour l'établissement de l'enquête, un questionnaire a été effectué et rempli lors des passages au niveau des élevages en question. L'entretien a été élaboré avec l'éleveur propriétaire ou le dirigeant de la ferme sous forme de questions directes, ou en choix multiples.

Ainsi, certaines données exploitées dans la présente étude proviennent des enquêtes menées, d'autres concernant la production laitière sont issues des contrôles laitiers disponibles au niveau des exploitations. En outre le concentré commercial (VL18) a fait l'objet d'analyses de la composition chimique (matières sèche, minérale, azotées totales et grasses) afin de déterminer sa valeur énergétique à l'aide du logiciel INRAtion. 
Les informations collectées vont d'une part, nous permettre de caractériser les exploitations et d'autre part pour la réalisation de l'analyse typologique. En effet par rapport aux objectifs tracés par l'enquête, les variables ont été réunies en deux volets (structure, fonctionnement et conduite) (tableau 1).

\section{Démarche de l'analyse}

Le traitement des données a été entrepris par une analyse des correspondances multiples $(\mathrm{ACM})$ à l'aide du logiciel SPAD version 6.5 (Coheris-SPAD, France) effectuée sur l'ensemble des variables qui sont le résultat des réponses des éleveurs aux différentes questions de l'enquête. L'ACM est suivi d'une Classification Ascendante Hiérarchique (CAH) qui permet de regrouper les individus en différentes classes homogènes sur la base des modalités.

Sur 29 variables qualitatives indicatrices (à la fois structurelles et fonctionnelles), 12 variables actives ont permis de réaliser une analyse factorielle des correspondances multiples. Ces variables ont été regroupés en 4 thèmes (tableau 2). Les principaux axes factoriels sont conservés pour la classification hiérarchique. Celle-ci est basée sur les critères d'agrégation de Ward et fournit une arborescence qui est interprétée et soumise à une partition. Le résultat obtenu se présente sous la forme d'un dendrogramme, arbre de construction des classes à partir duquel il sera possible de définir les regroupements les plus intéressants. L'interprétation s'est faite sur les résultats graphiques et numériques obtenus sous le logiciel SPAD.

Les classes sont caractérisées par les variables actives en étudiant les valeurs-tests de chaque variable dans chaque classe. Ces valeurs exprimées en nombre d'écarts types, résultent d'une comparaison de la moyenne de la variable dans la classe par rapport à sa moyenne générale.

La dernière étape consiste à interpréter les classes issues de l'analyse multi variée en termes de typologie des systèmes d'élevage dans cette région d'étude. 


\section{RESULTATS}

\section{Description des axes factoriels par les modalités actives}

L'analyse a permis d'identifier le nombre d'axes à conserver. Nous recensons 39 axes au total. La faible part de variance expliquée sur les premiers axes est une caractéristique de l'analyse factorielle de correspondance multiple. Dans notre cas, il convient d'archiver les trois premiers axes qui expliquent plus de $24 \%$ de l'information et dont les valeurs propres à retenir doivent être supérieures à 0,2 selon le critère empirique de Kaiser d'où les 2 premiers axes factoriels qui expliquent $18,34 \%$ de l'information, soit respectivement $11,29 \%$ et 7,05 $\%$. On peut décider de ne retenir que les deux premiers axes (le premier plan factoriel) car il est compréhensible par l'œil (c'est un plan) et ne déforme pas trop le nuage (il explique $82 \%$ de l'inertie du nuage) (Lebart et al. 1995).

D'après les résultats de l'ACM démontrés par la figure 2, nous pouvons déterminer que le premier axe caractérise principalement la structure des exploitations et qui sont bien représentées par les modalités qui ont un $\cos ^{2}$ supérieur à $0,20:$ surface agricole utile (SAU), surface fourragère $(\mathrm{SAF})$, surface irriguée $(\mathrm{SI})$, équipement agricole (EQU AGR), nombre d'étables (NE) et effectif des vaches laitières (VL). Selon la figure 2, à droite, on peut déduire que cet axe distingue les exploitations bien équipées présentant un potentiel important que ce soit en terres agricoles (utiles, fourragères et irriguées), ou en effectif de vaches laitières et qui sont localisées principalement dans les deux zones médiane et sud et oppose à gauche, les exploitations de petite taille et les moins équipées de la zone Nord où la contrainte foncière est plus rencontrée. Cet axe peut être considéré comme celui distinguant les exploitations de grande taille et bien équipées de celles de petite taille et moins équipées.

La modalité la plus significative présentée par l'axe 2 est la race exploitée avec un $\cos ^{2}$ égale à 0,23 . Selon la figure 2 , cet axe peut être considéré comme l'axe qui distingue en bas, les 
élevages exploitant massivement des vaches à haut potentiel génétique $(\mathrm{M})$ et oppose en haut les exploitations où la race locale $(\mathrm{L})$ et la race croisées $(\mathrm{A})$ sont les plus présentes.

\section{Description des groupes des systèmes d'élevages identifiés}

Ce travail a été suivi d'une CAH sur les résultats de l'ACM. Le résultat obtenu se présente sous la forme d'un dendrogramme (figure 3). Trois groupes ont été distingués par la typologie.

Les principaux groupes sont présentés par la figure 4, on peut distinguer :

Groupe 1 (G1) : Ce groupe est constitué de 24 exploitations (classe 1/3) soit 26,4\% de l'effectif total enquêté. Ce sont des élevages de taille moyenne avec terre. La SAU est importante tandis que l'intégration fourragère est peu développée. La SAF est assignée à quelques hectares de jachères non-travaillées ou cultivées en quelques fourrages essentiellement composés d'Orge en vert et de la Vesce avoine. Bien que les élevages de ce groupe soient localisés dans le périmètre irrigué englobant les deux zones Sud et Médiane, l'irrigation ne couvre que peu de terres. Ces élevages sont assez bien équipés en matériels agricoles ou en étables abritant les vaches laitières. L'exploitation de la race à haut potentiel génétique composés essentiellement des deux races performantes Prim Holstein et la Montbéliarde. L'association de l'élevage à la céréaliculture est une pratique qui domine dans ce groupe.

Groupe 2 (G2) : constitué de neuf exploitations (2/3), soit 9,9\% de l'échantillon enquêté. Ce sont des élevages de grande taille avec terre avec intégration fourragère considérable. Il englobe une minorité des éleveurs les plus performants et qui sont localisés majoritairement dans la zone médiane présentant les plaines de la région d'étude. L'irrigation couvre une superficie plus importante que les autres groupes. La surface fourragère est composée de jachères non-travaillées où pousse l'herbe pâturable et de cultures fourragères qui sont 
introduites dans la rotation (Orge en vert, Vesce avoine). La Luzerne et le Sorgho sont introduits dans trois exploitations. La composition raciale est dominée principalement par la race introduite importée composé essentiellement de la race Prime Holstein. L'équipement en étable ou en matériels agricoles est important. L'association élevage- céréaliculture est aussi présente dans ce groupe.

Groupe 3 (G3) : c'est le groupe le plus important en nombre d'exploitations (58 soit 63,7\%) (3/3), englobant des exploitations de petite taille avec peu de terres. Ce sont des élevages à caractère traditionnel disposant très peu d'équipements. La contrainte foncière est rencontrée le plus dans la zone Nord (hors le périmètre irrigué), une zone montagneuse où l'élevage en hors sol est le plus pratiqué. La SAF est consacrée majoritairement aux jachères non cultivées. L’irrigation est très peu développé prévaut majoritairement dans la zone médiane. L'exploitation de la race locale (Brune de l'Atlas), est plus dominante.

\section{DISCUSSION}

\section{Description générales des élevages étudiés}

Les groupes identifiés détiennent majoritairement des exploitations laitières n'ayant pas d'autres productions bovines. Le nombre des vaches laitières est dominant dans l'ensemble des élevages (1427 VL), l'engraissement des jeunes bovins ne présente qu'une faible proportion (15\%), En revanche la vente des veaux de l'année sans engraissement constitue un revenu d'appoint utilisé généralement pour l'achat d'un stock d'alimentation pour le troupeau. Le même constat a été rapporté par Mamine (2014) dans la même région d'étude. Le mode de reproduction se base sur la saillie naturelle avec une absence exclusive d'insémination artificielle. La majorité des exploitants utilisent un taureau reproducteur de leur cheptel tandis que les autres n'en disposant pas, sollicitent le géniteur jugé bon d'une autre exploitation. Achemaoui et Bendahmen (2016) affirment que la conduite de la reproduction du cheptel Algérien, souvent mal maîtrisée, est caractérisée par un faible développement de 
l'insémination artificielle (8 \% seulement des élevages), avec en conséquence des performances en dessous des objectifs techniques et économiques.

Nous avons ainsi constaté que les animaux se trouvent dans des étables entravées en quasipermanence, avec l'absence presque exclusive des aires d'exercice. Dans la pluparts des élevages enquêtés, il est difficile de parler d'étable au sens propre du terme, se sont généralement des anciennes bâtisses clôturées en béton ou en terre dure datés depuis l'époque coloniale. Par ailleurs ce sont les grands élevages laitiers spécialisés qui détiennent des étables assez modernes, neuves et aménagés. Il est toutefois a signalé que la stabulation entravée favorise les problèmes de l'appareil locomoteur (boiteries) et accentue la nervosité des vaches surtout pour celles qui se trouvent dans un local étroit, mal aéré et sans aucune aire d'exercice (Kaouch et al. 2011). Nous avons ainsi noté que peu d'étables disposaient d'abreuvoirs automatiques, ce qui signifie que les vaches ne s'abreuvent pas à volonté, mais plutôt quand l'éleveur les détachent, en les conduisant au bassin d'abreuvement. L'eau constitue ainsi un facteur limitant à la production des vaches dans notre région d'étude. Boudon et al. (2013) ont déterminé que les quantités journalières d'eau bues par une vache laitière sont très variables, de quelques litres avec une herbe verte en situation tempérée à plus de 120 litres avec une ration sèche. Il est même recommandé de bien veiller à ce que les troupeaux aient toujours à disposition de l'eau de qualité nécessaire à leur abreuvement notamment pendant les périodes de fortes chaleurs.

\section{Caractéristiques et principaux enseignements des groupes identifiés}

Cette analyse typologique a permis d'identifier et caractériser des groupes d'élevage bovins laitiers dans la wilaya de Souk-Ahras (tableau 3). Les résultats ont révélé que le foncier, la taille du cheptel ainsi que la composition raciale sont les facteurs qui discriminent le plus les trois groupes identifiés. La dotation en terres agricoles est plus importante dans les deux premiers groupes localisés principalement dans le périmètre irrigué de la région d'étude, 
présentant respectivement les plaines et les hauts plateaux. Il est toutefois a signalé que les cultures fourragères présentes une activité secondaire pour la plupart des exploitations de ces groupes, bien que le deuxième groupe détient une part plus importante en SAF mais cela reste toujours insuffisant pour combler les besoins alimentaire du troupeau laitier. Benniou et Aurby (2009) ont démontré que la prédominance de la céréaliculture occupant plus de terres car jugée plus rentable est alors vu comme le résultat de stratégies de durabilité des exploitations agricoles. Il est à souligner que les aliments destinés aux animaux provenant en partie de ces système de culture (fourrages, résidus de récolte, jachères, etc.).

Nous avons constaté que, l'un des principaux obstacles à la production fourragère vient du climat. Cependant, les fortes chaleurs estivales et la variabilité interannuelle de la pluviométrie constituent un frein à la croissance des cultures et notamment des fourrages. A ceci s'ajoute l'absence d'une stratégie d'irrigation convenable, malgré l'importance du potentiel hydrique de la région d'étude, ce qui a rendu l'approvisionnement en fourrages verts encore plus difficile. Même constat pour le périmètre irrigué du Haut-Cheliff au Nord de l'Algérie où la valorisation du potentiel fourrager, passe d'abord par la résorption de la jachère et par la mobilisation de l'eau d'irrigation au profit des fourrages (Belhadia et al. 2014).

L'analyse typologique des exploitations enquêtées fait ressortir aussi que la plupart des élevages étudiés sont concentrés dans la zone Nord de la wilaya, une zone montagneuse où l'élevage bovin reste largement pratiqué de manière traditionnelle en absence des terres ou d'investissements pour la modernisation des équipements qui restent très onéreux. En effet de nombreux élevage laitiers sont en hors sol ou ne disposent pas de surface fourragères suffisantes ; d'où des apports de paille et de foin extérieurs à l'exploitation pour compléter l'aliment concentré pour faire face au faible rendement et diversification des fourrages. En fait l'élevage hors sol tant en Algérie qu'à la région d'étude est un phénomène qui a été 
accentué dans une certaine mesure par le dénombrement des domaines agricoles socialistes (DAS), qui s'est traduit par le partage des biens productifs entre exploitants, les uns bénéficiant de la terre, les autres héritant de bâtiments d'élevages sans terre.

En fait, les conditions des élevages dans la région d'étude sont très particulières, les terres impliquées dans la production fourragère dans l'ensemble des élevages étudiés, ne présentent que $22,24 \%$ de la SAU, proviennent principalement de la jachère et des chaumes de céréales. Cependant, la production est loin de satisfaire quantitativement les besoins d'alimentation du cheptel. En effet la ration de base des vaches est composé de paille céréalière et de foin généralement de vesce avoine. L’approvisionnement en aliments vert n'est possible que durant le printemps en pâturage sur des prairies naturelles (jachères non travaillés) ou artificielle (orge en vert et avoine). Le déficit fourrager est alors constaté dans la majorité des exploitations à l'exception de quelques-unes qui ont introduit le Sorgho et la Luzerne. A ceci s'ajoute, la pratique d'ensilage qui y est pratiquement absente dans la quasi-totalité des exploitations à l'exception de la ferme pilote Yousfi-Tayeb de la localité de Tifach (nordouest de Souk Ahras). Cette situation a rendu la production laitière fondée en grande partie sur l'alimentation à base de concentré qui constitue une contrainte au développement de ces élevages. Mouhous et al. (2014) affirment qu'en Algérie, le concentré représente $55 \%$ des dépenses alimentaires dans les élevages bovins laitiers. En effet la part du concentré est importante dans la ration des vaches laitières, elle présente une moyenne quotidienne de 7,42 $\pm 2,45 \mathrm{Kg}$ par vache pour couvrir une production moyenne de 9,81 litres soit 0,86 UFL par kg de lait. Ces aliments concentrés qui sont fournis généralement par le marché (VL 18) se composent de son de blé ou d'orge, tourteau de soja et maïs. Belkheir et al. 2015 ont démontré que l'utilisation excessive de concentré par les éleveurs comme critère d'augmentation de la production laitière, par défaut d'une disponibilité de fourrages verts de 
bonne qualité et en absence de rationnement peut avoir des effets néfastes d'une part sur la santé et la carrière de la vache et d'autre part sur la durabilité de la filière lait dans la région.

Malgré l'exploitation des vaches à haut potentiel génétique (Prim Holstein) essentiellement par le deuxième groupe, les performances productives ne se sont pas traduites par les rendements escomptés qui sont mêmes à des niveaux insuffisants. Belhadia (2009) affirme que l'engouement des éleveurs pour la race Holstein se justifie par la préoccupation à augmenter leur capacité de production laitière mais aussi par l'historique de ces races qui ont été introduites dès les premières années de l'indépendance. En fait, la productivité des vaches demeure faible, elle présente des valeurs qui se rapprochent dans les trois groupes. Ce constat revient d'une part à la politique Algérienne de l'intensification de la production de lait frais qui est fondée principalement sur l'importation anarchique de plusieurs pays de vaches à hautes potentialités laitières (Zaida 2016) et d'autre part, la mauvaise adaptation de ces races à nos conditions climatiques ainsi le déficit en aliment adéquat. Il est toutefois à signalé que la race locale (Brune de l'Atlas), malgré sa faible productivité, est aussi présente dans les élevages étudiés essentiellement dans le troisième groupe. Comparativement aux races importées, les races locales sont caractérisées par leur adaptation aux conditions difficiles du milieu. En effet, elles sont adaptées à la marche en terrains difficiles et aux variations des régimes alimentaires (Bendiab 2012). Ceci explique que les tentatives d'amélioration de cette race demeurent dans une phrase de tâtonnement, sans suivi.

L'analyse a démontré clairement que les grandes exploitations de deuxième groupe qui sont les mieux équipés en machines agricoles (tracteur, faucheuse, et bien d'autres machines) ou en étables abritant le troupeau laitier. En revanche le troisième groupe englobant des petits élevages peu productifs et qui sont les moins équipés. L'accroissement de productivité des élevages laitiers va de pair avec le développement de la motorisation et de la modernisation des machines agricoles, pour la traction comme pour la récolte des fourrages ; des bâtiments 
pour les animaux et pour stocker les fourrages et de la mécanisation de la traite (Beranger et Lacalbe 2014).

La main d'œuvre exprimé en (UTH) correspond au travail fourni par une personne à capacité normale de travail permanents ou saisonniers occupée à temps complet sur l'exploitation pendant une année, quel que soit le nombre d'heures travaillées (qui comprennent éventuellement les exploitants eux-mêmes ou des membres de leur famille) (Vilain 2008). Nous avons constaté que la main d'œuvre demeure de type familial dans l'ensemble des groupes. De ce fait la main d'œuvre permanente en dehors du cadre familial est très peu développée et ne présente que 22,82\%. Ceci présente un sérieux problème pour l'avenir des élevages qui reposent généralement sur des systèmes informels pour l'emploi des ouvriers occasionnels. Cette situation est due à la conjonction de plusieurs facteurs : cette main d'œuvre du secteur agricole est mal rémunérée et non affiliée à la sécurité sociale, en plus d'une absence de législation de droit de travail (Bouchakour 2016). En effet l'élevage bovin laitier est une activité délicate qui nécessite des éleveurs qualifiés et expérimentés (Belkheir et al. 2011). Nous avons ainsi remarqué que la majorité de la main d'œuvre n'est pas qualifié, exerçant cette activité par expérience seulement, ou des exploitants qui ont hérité le métier et qui contribent à la survie de leurs familles. Bouchakour (2016) affirme que l'Etat Algérien dépense un budget minime pour ce genre de formation, ce qui laisse le secteur peu développé et en arrière des nouvelles inventions dans le domaine agricole, par rapport à nos voisins marocains et tunisiens. Il est noté que la faiblesse de technicité chez les éleveurs de la région d'étude dans la maîtrise et la rigueur de la conduite du troupeau, de la rationalisation de l'alimentation fait que les progrès enregistrés restent limités. 


\section{CONCLUSION}

Notre étude a permis de dresser un constat de la réalité de la situation des élevages bovins laitiers dans la wilaya de Souk-Ahras réputée pour être l'un des plus importants bassins laitiers national. Trois groupes d'élevage ont été distingués essentiellement par le foncier, la taille du cheptel et enfin par la race exploitée. En dépit des résultats, l'analyse souligne que le déficit fourrager fait que le rendement du cheptel laitier est très faible. Une telle situation est expliquée par la dominance du caractère traditionnel existant dans la région d'étude essentiellement présenté par le troisième groupe qui ne dispose pas, en règle générale, des superficies agricoles et notamment fourragères. Par ailleurs, le reste des élevages bien que les mesures d'appui sont plus importantes en matière de terres, taille et performance génétique du troupeau et en équipements agricoles, la productivité reste loin des objectifs d'un système performant et intensif. En effet ces exploitations pratiquent les cultures fourragères, mais partiellement, car elles consacrent la plus grande part des terres à la céréaliculture jugé plus rentable. A ceci s'ajoute le problème d'irrigation, qui est peu mobilisée en faveur du secteur agricole et fourrager plus particulièrement, malgré les potentialités hydriques confirmées de la wilaya. Nous avons ainsi constaté que la composition raciale du cheptel diffère d'un groupe à un autre. En effet, la race introduite importée à haut potentiel génétique est très présente dans l'ensemble des groupes, essentiellement le deuxième, mais cela malheureusement n'a pas contribué à l'amélioration de la productivité. Ce qui traduit la mauvaise adaptation de ces races à nos conditions de production essentiellement, une mauvaise alimentation à base de fourrages secs de mauvaise qualité et une rareté dans la diversification des fourrages verts.

Devant ce constat, les contraintes et les dysfonctionnements qui frappent nos élevages laitiers démontre que l'Etat est appelé à soutenir la production locale pour répondre aux besoins de la 
population en lait qui ne cesse d'augmenter et la mise en place d'un véritable plan de développement qui prenne en charge l'ensemble des activités liées à la filière.

En définitif, le développement de l'élevage bovin en Algérie est conditionné par l'amélioration des ressources fourragères. Ceci passe d'abord par le développement de la production de semences de qualité capables de s'adapter à notre climat et de la production de l'ensilage ainsi la mobilisation de l'eau d'irrigation au profit de ces fourrages. Toutefois il faut y joindre la production de génisses, pour le maintien d'un cheptel performant, réduisant ainsi le recours à l'importation anarchique du cheptel, et l'intensification de l'amélioration de la race locale. La vulgarisation agricole doit se focaliser sur des méthodes de gestion et de suivi technique pour l'amélioration de la qualification des exploitants. Celles-ci tentent de s'adapter aux contraintes socio-économiques du payé et aux aléas inhérents à leur approvisionnement en facteurs de production.

\section{REFERENCES}

Achemaoui, A., Bendahmane, M. 2016. Analyse des paramètres de reproduction dans un élevage privée à vocation Bovins laitiers au niveau de la wilaya de Sidi Bel Abbés. Revue Nature \& Technologie. B- Sciences Agronomiques et Biologiques, $14: 20$ - 22.

ANIREF. 2011. Agence Nationale d'Intermédiation et de Régulation Foncière Rubrique Monographie Wilaya e SOUK AHRAS, 8 P. $\underline{\text { www.aniref.dz/monographies/ar/soukahras.pdf }}$

Belhadia, A., Yakhlef, H., Bourbouze, A., Djermoun, A. 2014. Production et mise sur le marché du lait en Algérie, entre formel et informel. Stratégies des éleveurs du périmètre irrigué du Haut-Cheliff. Revue NEW MEDIT N. 1/2014, 41- 49.

Belkheir, B., Benidir, M., Bousbia, A., Ghozlane, F. 2011. Typologie des exploitations bovines laitières en zone de montagne de la région de Tizi-Ouzou (Algérie) Livestock Research for Rural Development .23 (3) : 2011. http://www.lrrd.org/lrrd23/3/belk23054.htm 
Belkheir, B., Ghozlane, F., Benidir, M., Benahmed, N., Agguini S. 2015. Production laitière, pratiques d'élevage et caractéristiques du lait en exploitations bovines laitières en montagne de Kabylie. Livestock Research for Rural Development 27 (8) 2015.

Bendiab, N. 2012. Analyse de la conduite d'élevage bovin laitier dans la région de Sétif. Mémoire de magister. Spécialité : Production Animale. Option: Amélioration de la Production Animale Université : Ferhat Abbas SETIF. Algérie : 129 p.

Béranger, C., Lacombe, PH. 2014. La recherche agronomique et la révolution agricole de la seconde moitié du XXe siècle : l'exemple de la prairie », Histoire de la recherche contemporaine, Tome III - N² | 2014, 167-179. journals.openedition.org/hrc/831

Bouchakour, R : 2016. L'impact de PNDA sur l'emploi agricole : cas de la pluriactivité des ménages agricoles en Algérie. Thèse doctorat en sciences agronomiques. Ecole nationale supérieure agronomique de Sétif Algérie, 238p.

Boudon, A., Khlil-Arfa, H., Menard, J.L., Brunschwi, P., Faverdin, P. 2013. Les besoins en eau d'abreuvement des bovins laitiers : déterminismes physiologiques et quantification INRA Prod Anim. 26 (3) : 249-262. prodinra.inra.fr/ft/A90E2CC2-4AC0-4DB2-A6ED$\underline{81958 B 17 B 6 F 3}$

Bouzida, S., Ghozlane, F., Allane, M., Yakhlef, H., Abdelguerfi, A. 2010. Impact du chargement et de la diversification fourragère sur la production des vaches laitières dans la région de Tizi-Ouzou (Algérie). Revu Fourrages : 204, 269-27. www.afpf-asso.org/download.php?type $=1 \& i d=1819 \&$ statut

CNIS. 2016. Centre national de l'informatique et des statistiques des douanes. Algérie.

Delaby, L., Peyraud, J.L. 2009. Valoriser les fourrages de l'exploitation pour produire du lait. Revue Fourrages. 198 : 191-210. http:/www.afpf-asso.fr/files/fourrages/articles/198-Delaby.pd 
Delagarde, R. 2009. Outils et indicateurs pour calculer et concilier ingestion des vaches laitières et valorisation de l'herbe au pâturage. Revue Fourrages. 198 : 175-190. www.afpf-asso.org/files/fourrages/articles/198-Delagarde.pdf

DSA. 2016. Direction des services agricoles de Souk-Ahras. Algérie.

Kaouche, S., Boudina, M., Ghezali, S. 2012. Évaluation des contraintes zootechniques de développement de l'élevage bovin laitier en Algérie : cas de la wilaya de Médéa. Revue « Nature \& Technologie ». $\mathrm{n}^{\circ} 06(85-92)$.

www.univ-chlef.dz/revuenatec/art_06_11.pdf

Leblanc, b. 2012. Analyse comparée des performances des systèmes de production des fermes laitières au canada et aux États-Unis. Mémoire de maîtrise en économie rurale. Département d'économie agroalimentaire et sciences de la consommation faculté des sciences de l'agriculture et de l'alimentation. Université Laval Québec. 132p.

www.cdc-ccl.gc.ca/cdc/userfiles/file/leblanc\%20benoit.pd

Lebart, L. Morineau, A. Piron, M. 1995. Statistique exploratoire multidimensionnelle. Edition Dunod, Paris. ISBN 210002886 3. 456p.

Mamine, F. 2014. Rationalité de l'informel : une analyse néo-institutionnelle de la filière lait á Souk-Ahras en Algérie. Thèse de Doctorat. École Doctorale Économie et Gestion de Montpellier - Spécialité : Sciences Économiques, 419p. www.univ-chlef.dz/revueNatec/issue-14/Article_B/Article355.pd

Mouhous, A., Alary, J., Huguenin, H .2014. Stratégies d'adaptation des éleveurs bovins laitiers en zone montagneuse d'Algérie Revue d'élevage et de médecine vétérinaire des pays tropicaux, 2014, 67 (4) : 193-200.

https://www.researchgate.net/.../316731461_Strategies_d'adaptation_des_eleveurs_bovin

Vilain, L. 2008. La méthode IDEA : Indicateurs de durabilité des exploitations agricoles. Troisième édition : Guide d'utilisation. Edition Educagri, $184 \mathrm{p}$. 
Zaida, W. 2016. Évaluation de la performance de la nouvelle politique de régulation de la production nationale de lait cru. Revue nouvelle économie. $\mathrm{N}^{\circ}: 15$. vol 02 (51-67).

\section{LISTE DES FIGURES}

Figure 1. Situation géographique de la wilaya de Souk-Ahras

Figure 2. Représentation des modalités actives sur les deux axes factorielles (facteurs 1 et 2)

Figure 3. Dendrogramme des groupes identifiés

Figure 4. Représentation graphique des trois groupes typologiques identifiés 


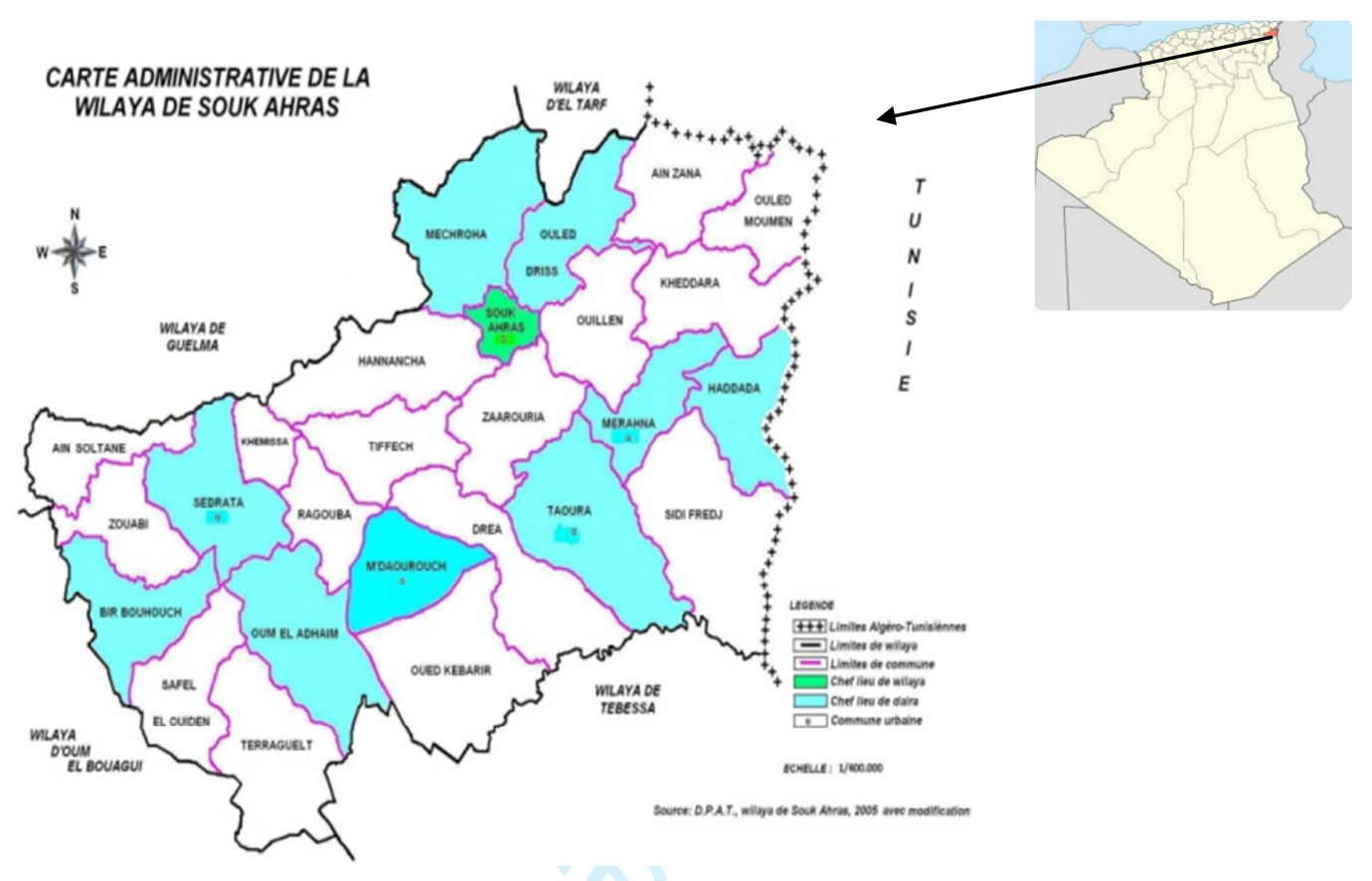

Fig.1. Situation géographique de la wilaya de Souk-Ahras (ANIREF, 2011) 


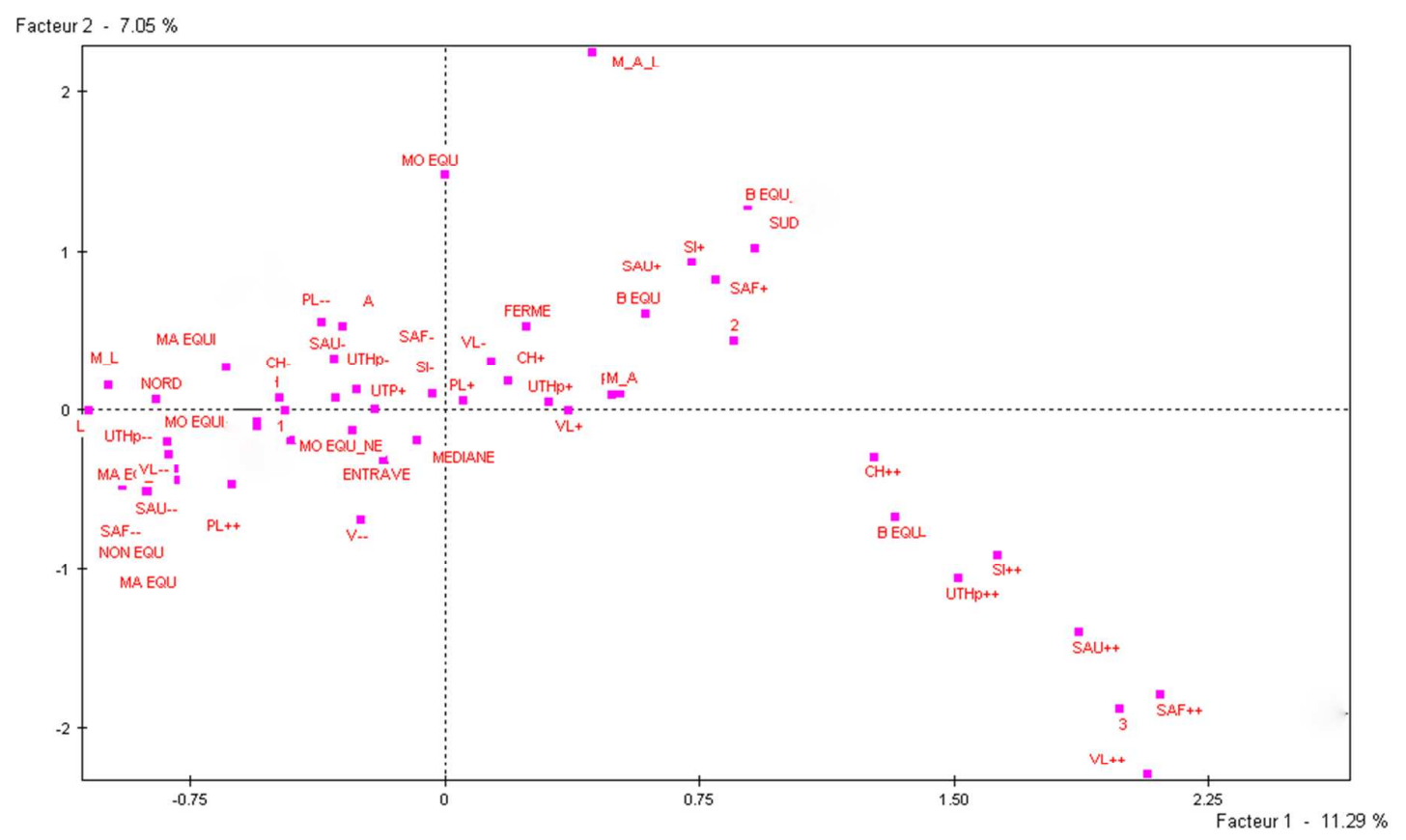

Fig. 2. Représentation des modalités actives sur les deux axes factorielles (facteurs 1 et 2)

Facteurs 1 et 2 : axes factoriels / Signes + , - sont utilisés pour désigner l'importance du caractère : ++ forte, + considérable, faible, - - négligeable / Races : $M$ (performante), L (locale), A (croisée) / $M_{-} A_{-} L$ (présence des trois races)/ Equipement : MO $E Q U$ (moyen), B EQU (bien équipé), NON EQU (Non équipé), $M A E Q U$ (mal équipé) 


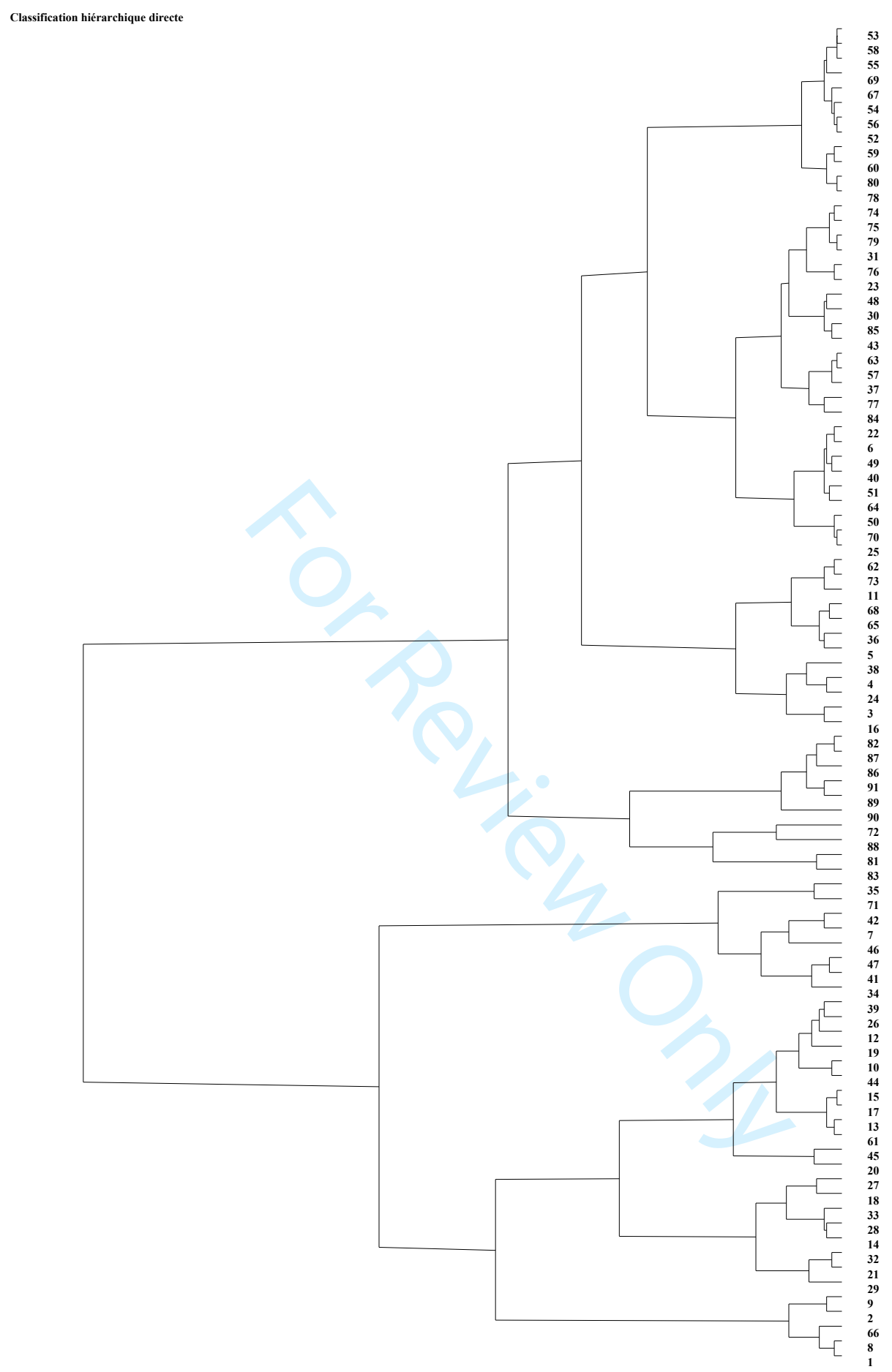

Fig. 3. Dendrogramme des groupes identifiés 


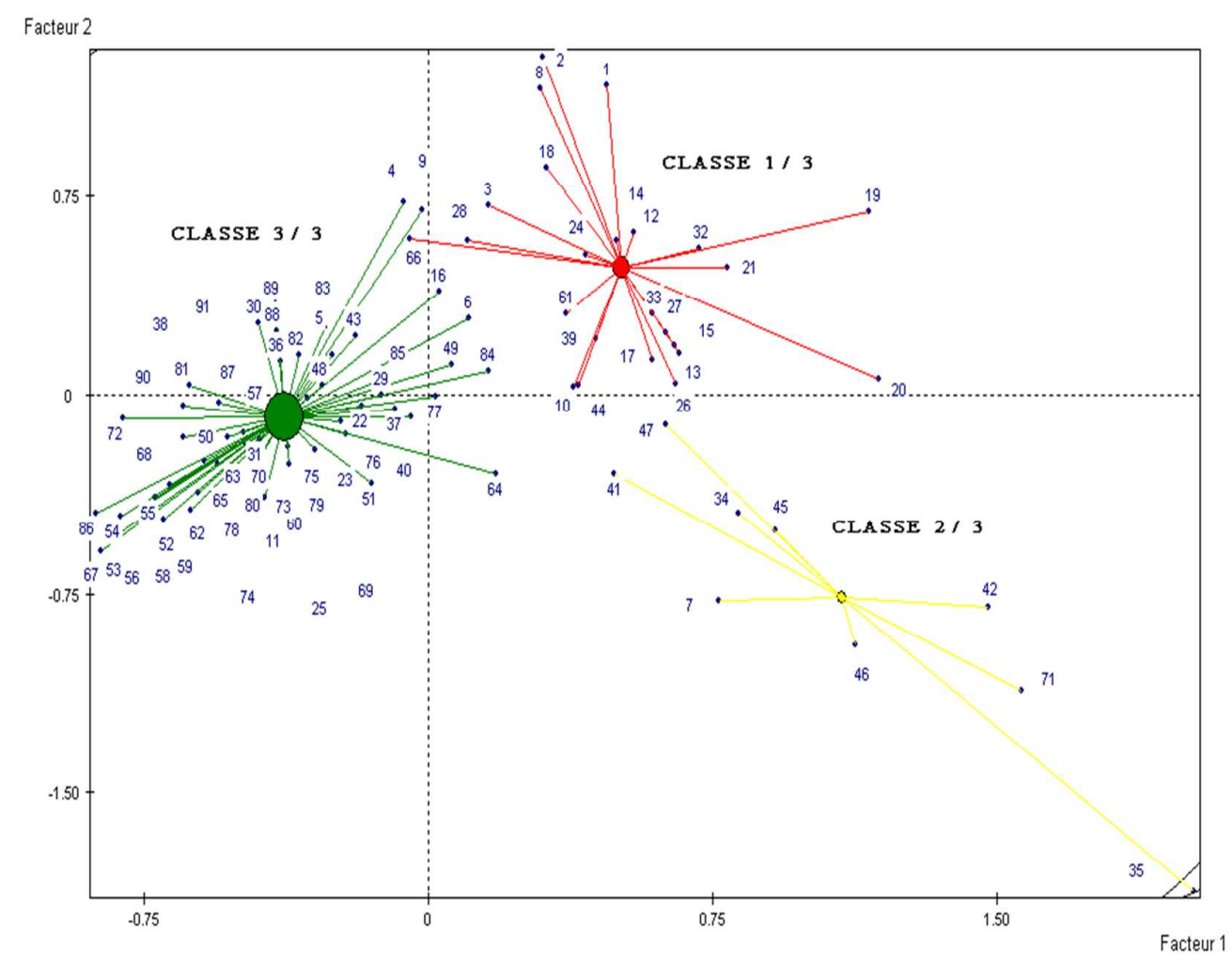

Fig. 4. Représentation graphique des trois groupes typologiques identifiés 
Tableau 1. Différents types de variables pour l'élaboration de l'enquête

\section{Types de variables}

\section{Structure de l'exploitation $\quad$ Fonctionnement et conduite}

*localisation géographique

*Superficie en propriété (ha)

*Superficie agricole utile (ha)

*Superficie agricole fourragère (ha)

*Surface irriguée (ha)

*Matériel d'irrigation (oui ou non)

*Nombre de chariot trayeur

\section{*Effectif vaches laitières}

*nombre de main d'œuvre familial

*unité de travail humain présente (UTHp)

*Niveau d'instruction de l'éleveur

*Formation dans le domaine de l'élevage
* composition de la ration de base

*Fréquence de distribution du concentré

*Quantité de concentré distribuée

$(\mathrm{Kg} / \mathrm{V} / \mathrm{J})$

*Production laitière (L/V/ans)

*Age au vêlage

*Gestion des vêlages

*Race

*Type de bâtiment

*Age du bâtiment

*Etat du bâtiment

* capacité d'accueil moyenne des vaches par bâtiment

*Moyenne de traite

*Hygiène de traite 
Tableau 2. Thèmes structurés à partir des questionnaires réalisés sur les exploitations

\begin{tabular}{|c|c|c|c|}
\hline Thèmes & $\begin{array}{c}\text { Nombre de } \\
\text { variables }\end{array}$ & Variables actives & $\begin{array}{l}\text { Nombre de } \\
\text { Modalités }\end{array}$ \\
\hline \multirow{5}{*}{$\begin{array}{l}\text { Foncier } \\
\text { agricole et } \\
\text { taille du } \\
\text { cheptel }\end{array}$} & 5 & Zone & 3 \\
\hline & & Superficie agricole utile (ha) (SAU) & 4 \\
\hline & & Superficie agricole fourragère (ha) (SAF) & 4 \\
\hline & & Superficie irriguée (ha) (SI) & 3 \\
\hline & & Effectif des vaches laitières (VL) & 4 \\
\hline \multirow[t]{5}{*}{ Equipements } & 5 & Matériel agricole (EQU) & 4 \\
\hline & & Nombre d'étables (NBR ETABLE) & 3 \\
\hline & & Capacité d'accueil des vaches dans l'étable $(\mathrm{CH})$ & 4 \\
\hline & & Type de bâtiment & 2 \\
\hline & & Unité de travail humain présente (UTHp) & 4 \\
\hline \multirow{2}{*}{$\begin{array}{l}\text { Performance } \\
\text { de production }\end{array}$} & 3 & Race & 5 \\
\hline & & Production laitière L/ vache/an & 4 \\
\hline
\end{tabular}


Tableau 3. Caractéristiques qualitatives et valeur moyenne des variables pour les différents groupes identifiés (valeurs en gras : valeur ayant permis la caractérisation des groupes)

\begin{tabular}{|c|c|c|c|}
\hline Variables & Groupe1 & Groupe2 & Groupe3 \\
\hline Superficie agricole utile (ha) & $167,33 \pm 124,76$ & $261 \pm 212,87$ & $36,22 \pm 24,72$ \\
\hline Superficie fourragère (ha) & $27,68 \pm 12,10$ & $56,66 \pm 27,54$ & $9,01 \pm 4,96$ \\
\hline Surfaces irriguées (ha) & $24,63 \pm 16,04$ & $17,77 \pm 27,28$ & $1,81 \pm 4,26$ \\
\hline Nombre de vaches (VL) & $15,37 \pm 4,44$ & $35,33 \pm 44,5$ & $12,75 \pm 7,88$ \\
\hline Race & $\begin{array}{l}\text { Introduite à haut } \\
\text { potentiel génétique }\end{array}$ & $\begin{array}{l}\text { Introduite à haut } \\
\text { potentiel génétique }\end{array}$ & Locale \\
\hline Equipements agricoles & Moyen & Important & Faible \\
\hline Nombre d'étable & $2 \pm 0,56$ & $3 \pm 0,76$ & $1,12 \pm 0,32$ \\
\hline Production laitière $(\mathrm{V} / \mathrm{J})$ & $2966,66 \pm 777,81$ & $3342,24 \pm 1135,47$ & $2845,83 \pm 1106,11$ \\
\hline Quantité de concentré $(\mathrm{Kg} / \mathrm{V} / \mathrm{J})$ & $7,68 \pm 2,43$ & $6,88 \pm 2,20$ & $7,58 \pm 2,74$ \\
\hline \multicolumn{4}{|l|}{ Mains d'œuvre présente } \\
\hline (UTHp) & $0,4 \pm 0,22$ & $0,54 \pm 0,23$ & $0,29 \pm 0,13$ \\
\hline
\end{tabular}


